\title{
Professional values: introduction to the theme
}

\author{
David Badcott
}

Published online: 11 September 2010

(C) Springer Science+Business Media B.V. 2010

We human beings not only confront values; we embody them, incarnate them, channel them. We are their transmitters, spectra dancing with their light. Joshua Loth Liebman, Peace of Mind, p. 167

A code of practice incorporating a declaration of professional values is both a distinguishing feature and its observance a condition of membership of most professional bodies. It is in effect a summary of important aspects of how members of that body are expected to behave and informs not only the membership itself, but also interested others and the general public. Within the values espoused in such codes, we should expect to be able to identify both generic values, that is those that reflect more general commitments to for instance, honesty, integrity, confidentiality and trustworthiness, but also values that relate directly to the nature of that particular profession or related professions. Thus for healthcare professionals, those values associated with beneficence, respect for autonomy and the centrality of patient care are prominent.

The group of papers that follows, evolved from a seminar on professional values in health care held at Cardiff University in August 2007 during the annual European Society for Philosophy of Medicine and Health Care conference. They do not represent a comprehensive summary or review of the values expressed in the various healthcare professionals" codes - these are readily available in the publications of the respective professional bodies. The intention of the papers is rather to highlight aspects that reflect either an intrinsic process of evolution in professional values, or a

D. Badcott $(\bowtie)$

Centre for Applied ethics, Cardiff University, Humanities

Building, Colum Drive, Cardiff CF10 3EU, UK

e-mail: badcottd@cf.ac.uk response to changing external demands, both welcome and unwelcome.

The relevance of reflexive reasoning is a crucial aspect of a profession that is explored by Edgar (2010) who draws on the example of British artists who successfully began a process of transition from trade to profession at the end of the 18th century. Edgar argues that to establish a profession requires not only a theoretically-informed educational base but also should incorporate processes of on-going reflection on professional objectives and just what it means to be a member of that profession. For artists, unlike craftsmen, a search for beauty cannot be codified or defined prior to its realisation. It is asserted that it is this vital element of reflexivity that distinguishes professions including medicine and other healthcare disciplines from occupations dependent on craft skills alone and for which instrumental reasoning is adequate. But paradoxically, a professional risks being disenfranchised from the members of the public he or she serves who are not party to this reflexive element and thus may not share a common vision of what constitutes a satisfactory outcome.

Sellman (2010) highlights the tension between institutional (managerial) and nursing (professional) practices in the UK, cautioning that nursing values, and particularly those associated with patient care are under threat from managerial controls. These threats are a consequence of a struggle by managers to accommodate severely constrained financial and human resources in a target-driven culture. Such drives for economic efficiency risk compromising core nursing values. For Sellman, as with Edgar, there is something over and above the mere mastery of technical skills that roughly equates with the internal goods of nursing. Drawing on the work of MacIntyre and others, Sellman argues that a MacIntyrean concept of (external) goods of effectiveness and (internal) goods of excellence 
provides a valuable perspective in which practitioners can appreciate the importance of holding firm on the latter which reflect core principles such as being truthful, just and courageous but does not lose sight of economic reality. Rather than a polarisation toward either effectiveness (target-related competence that diminishes the influence of values) or excellence (preoccupation with values that fails to address the reality of resource limitations), Sellman advocates seeking an Aristotelian mean that works toward a more equitable balance.

UK community pharmacists have experienced profound changes in the nature of their activities in recent years. Many of the traditional pharmacy craft-based dispensing skills have been superseded and the profession is moving progressively toward providing a more comprehensive professional therapeutic service, based on a concept of pharmaceutical patient care. An important aspect of the latter is the provision of help and advice to the community on a range of public health matters. These micro-level activities cover broadly, health education, health promotion and disease prevention and where possible are delivered to individuals, each with a particularised focus. In many respects, pharmacy has undergone a similar transition to that of art and from being largely a craft-based occupation to that of an important healthcare profession. And a significant part of that transition has been recognition of the values important to sustain and develop that professional status. In a review of some of the underlying factors, Badcott (2010) envisages the modern profession of pharmacy as being clearly situated within the framework of a vulnerability model of beneficence, recognition and respect. Key to this is the emphasis placed on the relationship between patient and healthcare practitioner, and one that reflects practice-related moral obligations. The professional values necessary to reflect the extension of pharmaceutical care to include micro-level public health activities are fully consistent with a vulnerability model.

But health care is not restricted to the traditional professions of medicine, nursing and directly allied activities. A wide range of complementary and alternative medical procedures is now available to members of the public as supplementary therapies or dissatisfied with conventional treatments. Some of these such as osteopathy, chiropractic and acupuncture have developed clear professional identities with formally recognised courses of higher educational study and statutory self-regulation. Tyreman (2010) in seeking to identify values that distinguish complementary and alternative therapeutic practitioners from those involved with conventional medicine recognises that considerations of patient autonomy and informed consent are key components of all health care practice whether conventional or not. What generally distinguishes complementary and alternative therapists is their adoption of a more holistic approach to patient well-being, restoring function and in particular a greater focus on outcomes that take account of the patient's desires and expectations. Additionally, complementary and alternative practitioners place great emphasis on natural remedies or advocate the value of adopting a more natural lifestyle, though neither concept is without difficulties in achieving a wholly meaningful definition or in a fully substantiated rationale. But it is perhaps in personally adopting "a more natural life-style" and in appreciating the benefits of holistic considerations in their own lives that complementary and alternative medical practitioners are able to actualise values that are not generally notable in conventional therapy.

At the end of the day, for all of us, the values espoused both in our domestic lives and in professional practice reveal a very personal spectrum, reflecting the varied influences and emphases of cultural background, education, practical training and experience. What is important for the individual is that there should be little or no dissonance between personal values in private life and professional values in healthcare practice. If the adoption of a set of professional values is a condition of membership of that profession, then those values must become an integral part of both professional practice and private life. Genuine values - those that are truly valued by their possessor are not disposable, to be taken up with a white coat or uniform and set aside at the end of the working day.

\section{References}

Badcott, David. 2010. Professional values in community and public health pharmacy. Medicine, Health Care and Philosophy. doi: 10.1007/s11019-010-9281-0.

Edgar, Andrew. 2010. Professional values, aesthetic values, and the ends of trade. Medicine, Health Care and Philosophy.

Liebman, Joshua Loth. 1946. Peace of mind: Insights on human nature that can change your life. New York: Simon \& Schuster.

Sellman, Derek. 2010. Professional values and nursing. Medicine, Health Care and Philosophy.

Tyreman, Stephen. 2010. Values in complementary and alternative medicine. Medicine, Health Care and Philosophy. 